\title{
A finite-volume gas-kinetic method for the solution of the Navier-Stokes equations
}

\author{
M. Righi \\ rigm@zhaw.ch
}

Zurich University of Applied Sciences

Winterthur, Switzerland

\section{ABSTRACT}

Gas-kinetic theory is also valid in the continuum regime: the Euler and Navier-Stokes equations can be obtained as projection of the Boltzmann equation on to the physical space $(x, t)$. The numerical schemes derived from gas-kinetic theory are computationally more expensive than Navier-Stokes based ones, but offer advantages which have been attracting a growing level of attention: they can (i) accommodate discontinuities at cells interface, (ii) provide high-resolution fluxes, (iii) provide advantages in the simulation of turbulence, $(i v)$ handle hypersonic and / or rarefied flows. This study extends the validation of gas-kinetic schemes investigating a few turbulent flow cases. At a slightly higher computational cost, gas-kinetic schemes provide results comparable to those obtained with well-validated Navier-Stokes schemes using the same turbulence model, grid and reconstruction order. In the case of shock-separated flows, the results obtained with the gas-kinetic scheme are even closer to experimental data. These findings are consistent with the idea that gas-kinetic theory is a physically more consistent framework for investigating the mechanics of fluids.

\section{NOMENCLATURE}

$a_{i}, A_{i} \quad$ expansion coefficients used for probability density function $\operatorname{erfc}^{i} \quad$ error function standard function in most programming languages

$f \quad$ probability density function 
$f_{0} \quad$ initial value (relative to a time step) of probability density function

$g \quad$ probability density function of a flow in thermodynamic equilibrium

$k \quad$ Boltzmann constant

$m \quad$ molecular mass

$p \quad$ pressure

$u_{\tau} \quad$ friction velocity

$y_{1}^{+} \quad$ distance from the boundary of the centroid of the first comp. cell, expressed in wall units

$B G K \quad$ Bhatnagar-Gross-Krook model of the Boltzmann equation

$C \quad$ scalar coefficient used in the definition of relaxation time

$C F L \quad$ Courant-Friedrichs-Lewy number

E total energy

EARSM Explicit Algebraic Reynolds Stress Model

GKS gas-kinetic scheme

$H(u) \quad$ Heaviside function

$K \quad$ turbulent kinetic energy

Kn Knudsen number

$L U-S G S$ Lower-Upper Symmetric Gauss-Seidel factorisation

$\mathrm{Ma} \quad$ Mach number

$N \quad$ effective degrees of freedom of the flow molecules

NS Navier-Stokes scheme

$P \quad$ turbulent production

RANS Reynolds-Averaged Navier-Stokes

Re Reynolds number

$S_{i, j} \quad$ strain rate tensor

$T \quad$ temperature

$U \quad$ velocity

WENO weighted-essentially-non-oscillatory reconstruction

\section{Greek symbols}

$\beta, \beta^{*}, \hat{\gamma}, \gamma^{*}, \sigma, \sigma^{*}$ scalar coefficients used in turbulence model

$\gamma$

$\delta_{i j}$

$\lambda$

$\mu$

$\mu_{t}$

$v$

$\xi$

$\rho$

$\tau$

$\tau_{t}$

$\tau_{i j}$

$\tau_{w}$

$\omega$

$\Delta_{t}$

$\Psi$ specific heat ratio

Kronecker delta function

$\mathrm{m} / 2 \mathrm{Kt}$

molecular viscosity

turbulent viscosity

kinematic viscosity

internal degrees of freedom of molecules

density

relaxation time in BGK model

turbulent relaxation time in BGK model

turbulent stresses tensor

wall shear stress

turbulent specific dissipation

time step length

$\left\{1 u_{i 2}^{1}\left(u_{i}{ }^{2}+\xi^{2}\right)\right\}^{T}$ 


\subsection{INTRODUCTION}

Numerical schemes based on the Navier-Stokes (NS) equations have for many years benefited from the valuable work of mathematicians and engineers. Aerodynamicists dispose of accurate and fast simulation tools, which can be applied to complex geometries and challenging flow conditions, providing physically consistent results in many cases.

However, limitations still apply. The simulation of complex flow cases might show a significant dependence on the numerical scheme chosen or on the turbulence model and in some cases these two factors have a reciprocal influence. A further limitation comes from the unsuitability of the Euler and Navier-Stokes equations to simulate local or global rarefaction; the simulation of shocks strongly relies for instance on numerical dissipation and not on the resolution of the shocklayer.

The gas-kinetic theory, largely based on the Boltzmann equation and its derivatives, provides a more physically consistent representation of fluid mechanics than the Navier-Stokes equations. It is well known that Navier-Stokes and Euler equations can be derived from the Boltzmann equation for instance by means of the Chapman-Enskog expansion ${ }^{(1)}$. Numerical schemes based on gas-kinetic theory or Gas-Kinetic Schemes (GKS) might therefore help in overcoming limitations of Navier-Stokes based methods. Moreover, gas-kinetic can also handle in a more natural way the numerical discontinuities that are an essential ingredient of all Godunov-type schemes ${ }^{(2)}$, including high resolution ones.

A further potential advantage of GKS concerns the simulation of turbulent flows. Virtually all modern turbulence modelling techniques rely on the concept of eddy viscosity, originally developed by Lord Kelvin and Osborne Reynolds, and based on the similarity between the distribution of eddies and the distribution of the molecules in a fluid. However, Navier-Stokes based methods, are unable to exploit this similarity. GKS, unlike Navier-Stokes based methods, model the turbulent flow as a distribution of eddies interacting with one another ${ }^{(3-5)}$.

The ambition and originality of this study concern the modification of a GKS in order to include the effect of turbulence in the numerical fluxes. A standard linear two-equation turbulence model is used to model turbulence. It is important to note that the use of turbulence models with gas-kinetic schemes is still relatively unexplored, whereas they are commonly used with Lattice Boltzmann methods $^{(3)}$. The scheme used is the GKS developed by Xu in $2001^{(6)}$, also investigated by other researchers $^{(7,8)}$. It has shown remarkable reliability and accuracy in a number of cases, ranging from viscous subsonic flows to hypersonics.

The cases chosen for the study include popular benchmarks characterised by shock-boundary layer interaction. The criteria used to assess the behaviour of the GKS are the capability to predict the shock's position and strength with respect to a traditional Navier-Stokes scheme.

This paper presents a brief description of the GKS together with the modelling of turbulence, followed by the details of the numerical implementation and the results of the numerical simulations.

\subsection{GAS-KINETIC SCHEME}

In the 1990s a few gas-kinetic schemes for the Euler and the Navier-Stokes equations had been developed and put forward ${ }^{(6-11)}$ as an alternative to the traditional schemes.

Broadly speaking, GKS replace the Riemann solver and the linear viscous fluxes with a different, and physically more consistent, process based on the Bhatnagar-Gross-Krook (BGK) model of the collision term in the Boltzmann equation, whereas the Riemann solver assumes an infinite number of collisions. GKS are therefore able to investigate shock layers and hypersonic flow, where the number of collisions in a computational cell cannot be considered infinite. However, 
GKS also provide advantages in the hydrodynamics limit.

The main idea behind these schemes is to consider a discontinuous state across interfaces, re-construct the equilibrium and non-equilibrium distribution functions based on the macroscopic flow variables and calculate the evolution of the distribution functions during a time step $\Delta_{t}$ integrating the BGK-Boltzmann equation. The macroscopic flow quantities are then recovered taking moments of the solution distribution function.

The advantages with respect to more traditional approaches are $(i)$ the treatment of discontinuities in a natural way, (ii) the coupling of the spatial and the temporal evolution of the gas during a time-step , (iii) combined advective and viscous fluxes and (iv) a formulation that clearly separates physical and artificial dissipation.

The macroscopic variables $\rho, U=\left[u_{1} u_{2} u_{3}\right]^{T}$, and $E$ are used to describe density, velocity and total energy of a gas. Instead of using the well-known Navier-Stokes equations, the BGK model is written following ${ }^{(12)}$ :

$$
\frac{\partial f}{\partial t}+u_{i} \frac{\partial f}{\partial x_{i}}=\frac{(g-f)}{\tau},
$$

where the summation convention holds, $f$ is the gas distribution function, $g$ is the equilibrium state, a Maxwellian distribution, approached by $f$ and $\tau$ is the particles collision time, which is related to the molecular viscosity and heath coefficients of the gas. The variable $\xi$ is related to the additional degrees of freedom of the gas molecules. $\xi$ has $N$ degrees of freedom, where:

$$
N=\frac{3-3 \gamma}{\gamma-1}+1 \text {, }
$$

where $\gamma$ is the specific heat ratio. The equilibrium distribution is:

$$
g=\rho\left(\frac{\lambda}{\pi}\right)^{\frac{N+2}{N}} e^{-\lambda\left(\left(u_{i}-U_{i}\right)^{2}+\xi^{2}\right),}
$$

where $\lambda=m 2 k T, m$ is the molecular mass, $k$ is the Boltzmann constant, and $T$ is temperature. The relation between macroscopic variables and gas distribution function is:

$$
\left\{\begin{array}{c}
\rho \\
\rho u_{i} \\
\rho E
\end{array}\right\}=\int \psi f \mathrm{~d} \Xi,
$$

where $\Psi$ is:

$$
\psi=\left\{\begin{array}{c}
1 \\
u_{i} \\
\frac{1}{2}\left(u_{i}^{2}+\xi^{2}\right)
\end{array}\right\} .
$$

Note that $\mathrm{d} \Xi=\mathrm{d} u_{1} \mathrm{~d} u_{2} \mathrm{~d} u_{3} \xi^{N-1} \mathrm{~d} \xi$. Conservation of mass, momentum and energy during particle collision is expressed by:

$$
\int(g-f) \psi \mathrm{d} \Xi=0
$$

The BGK model shown in Equation (1) has an analytical solution: 


$$
f(x, y, z, t, u, v, w, \xi)=\frac{1}{\tau} \int_{0}^{t} g\left(x^{\prime}, y^{\prime}, z^{\prime}, t, u, v, w, \xi\right) e^{-\left(t-t^{\prime}\right) / \tau} \mathrm{d} t^{\prime} e^{-t / \tau} f_{0}(x-u t, y-v t, z-w t),
$$

where $f_{0}$ is the initial gas distribution function, $x^{\prime}=x-u\left(t-t^{\prime}\right) ; y^{\prime}=y-v\left(t-t^{\prime}\right) ; z^{\prime}=z-w\left(t-t^{\prime}\right)$. The kernel of the GKS consists in expressing the distribution function $f$ at cells or volumes interfaces in order to assess the fluxes as functions of $f$. For instance the flux in direction $i$ at the interface between cells $n$ and $n+1$ can be expressed as a first moment of $f$ :

$$
F_{i}^{n+1 / 2}=\int_{0}^{\Delta t} \int u_{i}^{n+1 / 2} \psi^{v+1 / 2} f\left(x^{v+1 / 2}\right) \mathrm{d} \Xi
$$

The distribution functions $f_{0}$ and $g$ in Equation 7 must be consistent with the macroscopic variables and their gradients. An important assumption in the derivation of the GKS is that whereas equilibrium distributions are Maxwellians, the nonequilibrium distribution are expressed as Taylor expansion of Maxwellian distribution. Assuming an interface normal to direction 1 located at $x_{1}=0$, the initial equilibrium distribution is expressed as:

$$
g=\left\{\begin{array}{c}
g_{0}\left(1+a_{i}^{-l} x_{i}-\bar{A} t\right) x_{1}<0, \\
g_{0}\left(1+a_{i}^{-r} x_{i}-\bar{A} t\right) x_{1}<0,
\end{array}\right.
$$

where $g_{0}$ is a Maxwellian derived from a state $\left[\rho_{0} \rho u_{0 i} \rho_{0} E_{0}\right]$. The initial distribution $f_{0}$ can be expressed as:

$$
f_{0}=\left\{\begin{array}{c}
g^{l}\left(1+a_{i}^{l} x_{i}\right)-\tau\left(a_{i}^{l} u_{i}+A^{l}\right), x_{1}<0, \\
g^{r}\left(1+a_{i}^{r} x_{i}\right)-\tau\left(a_{i}^{r} u_{i}+A^{l}\right), x_{1}<0,
\end{array}\right.
$$

where $g^{l}$ and $g^{r}$ are Maxwellian distribution on both sides of the interface, which are indicated as left and right. The choice of the terms used in the expansion in Equation 10 is critical for the type of GKS. The terms proportional to $\tau$ represent the non-equilibrium parts in the ChapmanEnskog expansion ${ }^{(1)}$, whereas the expansion in the spatial directions $x_{i}$ is directly related to the formal accuracy of the resulting scheme. Moreover, one can have a directional splitting scheme by simply expanding in the direction normal to the interface, or a truly multi-dimensional scheme by considering the derivatives in all directions.

Each of the coefficients in Equations (9) and (10) is expanded as:

$$
a_{i}=a_{i 1}+a_{i 2} u+a_{i 3} v+a_{i 4} w+a_{i 5}\left(u^{2}+v^{2}+w^{2}+\xi^{2}\right) \text {. }
$$

All the components of the coefficients are determined from compatibility relations with the macroscopic variables and the conservation condition in Equation (6). The details can be found in Ref. 6. The determination of all coefficients involves the solution of numerous (depending on the dimensions) linear systems and the evaluation of the erfc function, which contribute to the computational cost. Inserting Equations (9) and (10) into the Equation (7), one obtains $f$ :

$$
\begin{aligned}
& f=\left\{\left[\bar{A} t+1-e^{-t / \tau}\left(a_{i}^{-1} u_{i} h^{l}+a_{i}^{-r} u_{i} h^{r}\right)\right] g_{0}-t e^{-t / \tau}\left(a_{i}^{1} u_{i} h^{l} g^{l}+a_{i}^{r} u_{i} h^{r} g^{r}\right)\right\} \\
& +\tau\left\{\left(-1+e^{-t / \tau}\right)\left[\bar{A}+h^{l} \bar{a}_{i}^{l} u_{i}+h^{r} \bar{a}_{i}^{r} u_{i}\right] g_{0}-t e^{-t / \tau}\left[\left(a_{i}^{1} u_{i}+A^{l}\right) h^{l} g^{l}+\left(a_{i}^{r} u_{i}+A^{r}\right) h^{r} g^{r}\right]\right\},
\end{aligned}
$$


where $h^{1}=H(u)$ and $h^{r}=1-H(u)$ and all coefficients are intended as series expansions in the form of Equation (11). The terms in the first line of Equation (12) are the ones generating the advective fluxes whereas the ones in the second line of Equation (12) originate the viscous fluxes. In both parts one can see a first contribution from the equilibrium distribution $g_{0}$ and a second one from the initial discontinuous distribution $f_{0}$, composed by $g^{1}$ and $g^{r}$. The terms proportional to $g_{0}$, a BGK-model compatible flow state on the interface, generate fluxes comparable to the ones from central differences, whereas the ones depending on $g^{1}$ and $g^{r}$ generate fluxes similar to the ones from upwind schemes.

Similar considerations have led to schemes for the Euler equations. The importance of Equation (12) is not limited to the possibility to derive an upwind scheme directly from the BGK model, but also to provide a much richer description of the underlying physics, as one can infer from the observation of the viscous terms and from the fact that the relative weights used in the combination also depend on the relaxation time $\tau$. The fluxes can be evaluated inserting Equation (12) into Equation (8). It is interesting to note that GKS fluxes are second-order accurate in time; the fluxes are formally an expansion in $\Delta t$ truncated at the second $\operatorname{order}^{(5)}$. Time accurate fluxes also mean that the spatial and temporal evolution during a $\Delta t$ time step of the gas are coupled.

Non-equilibrium parts, i.e. all the terms proportional to $\tau$ affect fluxes in Equation (8) but have no contribution to macroscopic variables:

$$
\int\left(a_{i}^{l} u_{i}+A^{l}\right) g^{l} \mathrm{~d} X i=0, \quad \int\left(a_{i}^{r} u_{i}+A^{r}\right) g^{r} \mathrm{~d} X i=0,
$$

The relaxation time $\tau$ is defined as a function of the molecular viscosity, $\tau=\mu / p$ according to the kinetic theory. Artificial viscosity is added explicitly by including a term proportional to the pressure jump across the interface, qualitatively similar to the artificial dissipation used in the Jameson, Schmidt, Turkel scheme ${ }^{(13)}$.

$$
\tau=\frac{\mu}{p}+C \frac{\left|p^{r}-p^{l}\right|}{\left|p^{r}+p^{l}\right|} \Delta t,
$$

where $p^{l}$ and $p^{r}$ are the value of pressure on the left and right hand side of the interface. A known drawback of the BGK model is that it implies a unity Prandtl number; the heath flux must therefore be corrected for realistic gas/fluids ${ }^{(6)}$. The coefficient $C$ is determined heuristically; on average $C$ has been fixed at around $0 \cdot 5$. Refer to Ref. 7 for a more complete discussion on the role of artificial dissipation in gas-kinetic schemes.

\subsection{TURBULENCE MODELLING}

The present study is based on a simple modelling technique: the Reynolds approach, which explicitly resolves the mean flow and models the effects of all turbulent length scales - often referred to as RANS in its implementation with the Navier-Stokes equations. Turbulent quantities are modelled according to $k-\omega \operatorname{model}^{(14)}$, a well-known member of the two-equation class of turbulence models.

$$
\begin{aligned}
& \frac{\partial \rho K}{\partial t}+\frac{\partial \rho u_{j} K}{\partial x_{j}}=\mathrm{P},-\beta^{*} \rho \omega K+\frac{\partial}{\partial x_{j}}\left(\left(\mu+\sigma^{*} \mu_{t}\right) \frac{\partial K}{\partial x_{j}}\right), \\
& \frac{\partial \rho \omega}{\partial t}+\frac{\partial u_{j} \omega}{\partial x_{j}}=\hat{\gamma} \rho \frac{\omega}{K} P+\beta \rho \omega^{2}+\frac{\partial}{\partial x_{j}}\left(\left(\mu+\sigma \mu_{t}\right) \frac{\partial \omega}{\partial x_{j}}\right) .
\end{aligned}
$$


The effect of turbulence on the mean flow is modelled through eddy viscosity:

$$
\mu_{t}=\gamma^{*} \frac{\rho K}{\omega} .
$$

$P$ indicates the turbulence production, calculated exactly from mean flow gradients and the turbulent stress tensor:

$$
P=\tau_{i j} \frac{\partial u_{i}}{\partial u_{j}},
$$

$\tau_{i j}$ is the turbulent stress tensor which is modelled assuming proportionality to mean flow gradients through the eddy viscosity and adding the contribution of turbulent kinetic energy to the isotropic part:

$$
\tau_{i j}=\mu_{t}\left(2 S_{i j}-\frac{2}{3} \frac{\partial u_{k}}{\partial x_{k}} \delta_{i j}\right)-\frac{2}{3} / \rho K \delta_{i j},
$$

where $S_{i j}$ is the strain rate tensor:

$$
S_{i j}=\frac{1}{2}\left(\frac{\partial u_{i}}{\partial x_{j}}+\frac{\partial u_{j}}{\partial x_{i}}\right)
$$

The parameters $\beta, \beta^{*}, \hat{\gamma}, \gamma^{*}, \sigma, \sigma^{*}$ are the standard ones for the 2006 version of the $k$-w model ${ }^{(14)}$ :

$$
\beta=\frac{3}{40}, \beta^{*}=\frac{9}{100}, \hat{\gamma}^{*}=\frac{5}{9}, \gamma^{*}=1, \sigma=\frac{1}{2}, \sigma^{*}=\frac{1}{2},
$$

The implementation of a turbulence model into a gas-kinetic scheme is straight-forward: the BGK model in Equation (1) can be reexpressed as

$$
\frac{\partial f}{\partial t}+u_{i} \frac{\partial f}{\partial x_{i}}=\left(\frac{g-f}{\tau^{*}}\right),
$$

where the relaxation time $\tau_{*}$ includes both molecular and turbulent phenomena and can be expressed as a combination of molecular and turbulent relaxation times:

$$
\tau^{*}=\tau+\tau,
$$

where the molecular relaxation time $\tau$ is proportional to the molecular viscosity as in Equation 14 and $\tau_{t}$ is proportional to eddy viscosity:

$$
\tau_{t}=\frac{\rho K}{\omega p}=\frac{\mu_{t}}{p}
$$

Equation (24) can be re-expressed as: 


$$
\tau^{*}=\frac{\mu+\mu_{t}}{\pi}+\frac{\left|p^{r}-p^{l}\right|}{\left|p^{r}+p^{l}\right|} \Delta t,
$$

where artificial viscosity has been added in the same way as in Equation (14). A more accurate expression of the turbulent relaxation time can be derived from Ref. 3:

$$
\tau_{\tau}=\frac{\rho K / \omega}{p\left(1+\eta^{2}\right)^{1 / 2}},
$$

where $\eta=S / \omega$ and $S$ is a scalar representing local velocity gradient. Note that $\eta$ is a ratio between characteristic timescales: $\omega^{-1}$ can be seen as the timescale of the modelled turbulence, $S^{-1}$ can be related to the characteristic time of the mean flow. The role of $\eta$ in Equation 27 can be seen as a second order eddy viscosity correction, which becomes ignificant as soon as the timescale of modelled turbulence becomes significant with respect to the timescale of the mean flow. The final expression for the relaxation time $\tau^{*}$ becomes:

$$
\tau^{*}=\frac{\mu}{p}+\frac{\rho K / \omega}{p\left(1+\eta^{2}\right)^{1 / 2}}+C \frac{\left|p^{r}-p^{l}\right|}{\left|p^{r}+p^{l}\right|} \Delta t .
$$

\subsection{NUMERICAL SIMULATIONS}

All numerical simulations compare the results of a GKS and an NS scheme. Both schemes are implemented in a 2D structured, finite-volume spatial discretisation. The two schemes share the reconstruction of the conservative variables at cells interfaces but differ in the evaluation of fluxes and in time stepping. Whereas the NS fluxes are obtained from Roe's approximate Riemann solver (advective) and from central differences (viscous), the GSK fluxes are obtained from Xu's scheme $^{(6)}$ extended to multi-dimensions.

The NS scheme is advanced in time with a third order Runge Kutta method. The GKS uses a time-accurate single-step approach, despite the fact that most previous applications of GKS have often used conventional multistep techniques ${ }^{(7,8)}$. Both schemes use pre-conditioning (approximate LU-SGS based on the approximate factorisation of the NS operator ${ }^{(15)}$ plus local time-stepping and multigrid acceleration ${ }^{(16)}$. The approximate LU-SGS factorisation had already been used with the same GKS operator in Ref. 17. However, the reduction of the computational cost of GKS schemes is still largely an open issue. Most computations have been conducted with CFL $>5$.

The evaluation of GKS fluxes is computationally approximately three times more expensive. However, thanks to the second-order time accurate fluxes, the GKS requires less evaluations per time step and often requires less iterations to reach a given convergence level. On average, the GKS has requested approximately $30 \%$ to $50 \%$ more computational time.

\subsection{Laminar wake instabilities}

At low Reynolds numbers and low angle-of-attack the vortexes of the wake behind an aerofoil are shed in a laminar mode ${ }^{(18)}$ and can be numerically simulated without turbulence model. The flow behind a NACA 0012 aerofoil at Reynolds number (chord) of 20,000 and 30,000 at the angle-of-attack of 1 degree has been simulated with the NS and GKS schemes. Both solvers use a fifth order WENO reconstruction ${ }^{(19,20)}$ and are in this case used in time-accurate mode, i.e. with 


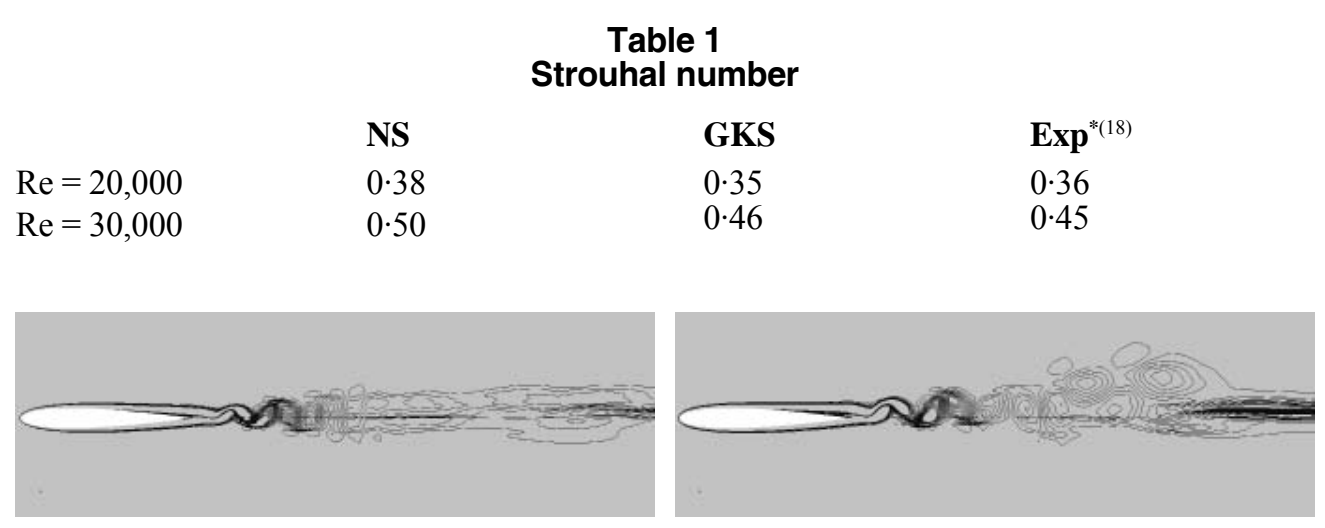

Figure 1. Vorticity contour (10 lines between -1 and +1 ) behind a NACA 0012 aerofoil at $1^{\circ}$ incidence, $\mathrm{Re}=30,000$, obtained from the NS solution (left) and from the GKS solution (right).

no pre-conditioning nor multigrid. GKS uses a multi-dimensional scheme. The computational mesh is of $C$-type with $432 \times 104$ cells.

The values of Strouhal number (based on aerofoil thickness) measured from the two simulations are summarised in Table 1. The GKS predicts a value which is closer to the experiments, the error in Strouhal number being approximately one third of that made by the NS scheme. Interestingly, the GKS provides a better resolution of the vortexes, as shown in Fig. 1, despite both schemes using the same reconstruction. This finding confirms that the GKS exploits the available information (the value of conservative variables and their gradients) in a more effective way than the NS scheme. This is consistent with the finding by $\mathrm{Li}$ and $\mathrm{Xu}^{(21)}$.

\subsection{RAE2822 and NACA 0012 aerofoil in transonic flow}

Cases 9 and 10 of the measurements conducted by $\operatorname{Cook}^{(22)}$ on the RAE2822 supercritical aerofoil as well as Harris' investigation ${ }^{(23)}$ of the transonic flow around the NACA 0012 aerofoil are arguably the most popular benchmarks for NS solvers and turbulence models, developed for transonic flow. In RAE2822 Case 9 the boundary layer does not separate, whereas in RAE2822 Case 10 and around the NACA 0012 at $\mathrm{M}=0 \cdot 800, \mathrm{Re}=9 \times 10^{6}$ and $\alpha=2 \cdot 83^{\circ}$ the shock-boundary layer interaction leads to a large flow separation on the upper side of the aerofoil.

Figures 2, 3 and 4 show the pressure distributions obtained with the NS and the GKS schemes in the three cases. Both schemes use second order reconstruction wi ${ }^{\circ}$ th van Leer limiter, approximate LU-SGS pre-conditioning and multigrid. NS scheme uses Roe's approximate Riemann solver and GKS uses a bi-dimensional scheme. The computational meshes are $C$-type with $625 \times 125$ cells (RAE 2822) and 624128 cells (NACA 0012), with a resolution enough to resolve the laminar sublayer in both cases, e.g. $y_{1}^{+}<1$, where $y_{1}^{+}$is the distance of the first computational point from the wall expressed in wall units. One wall unit corresponds to $v / u_{\tau}$, where $u_{\tau}=\sqrt{\tau_{w} / \rho}, \tau_{w}$ is the wall shear stress and $v$ is kinematic viscosity.

The accuracy of the results is assessed in terms of the position and thickness of the shock on the pressure coefficient chart. The results obtained from the NS and GKS schemes for Case 9 are shown in Fig. 2: both schemes predict a shock position at $68 \%$ of the chord with a thickness of $4 \%$ of the chord. These values are consistent with the ones found experimentally by Cook ${ }^{(22)}$. The differences between the predictions and the experimental data is of the same order of magnitude of the measurement uncertainty. 


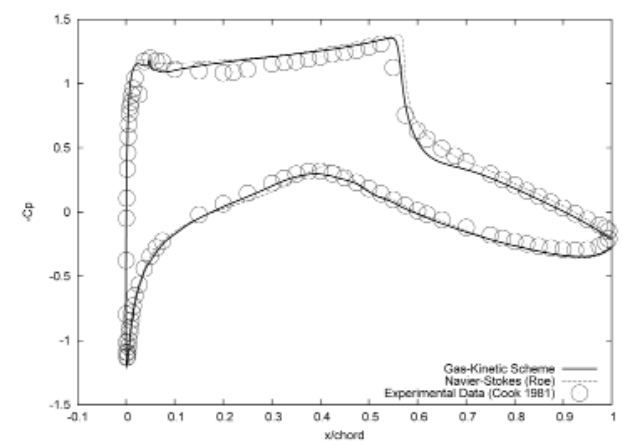

Figure 2. $\operatorname{RAE} 2822$ Case $9, \operatorname{Re}=6 \cdot 2106, M=0 \cdot 725$, angle-of-attack $\alpha=2 \cdot 30^{\circ}$ pressure coefficient.

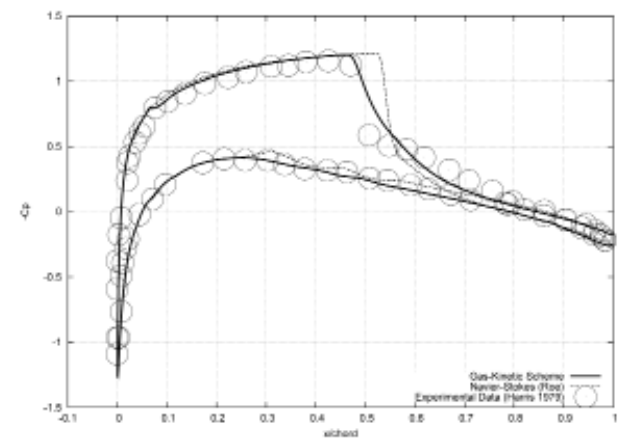

Figure 4. NACA 0012, $\mathrm{Re}=9 \cdot 0106, \mathrm{M}=0 \cdot 799$, angleof-attack $\alpha=2 \cdot 26^{\circ}$ pressure coefficient.

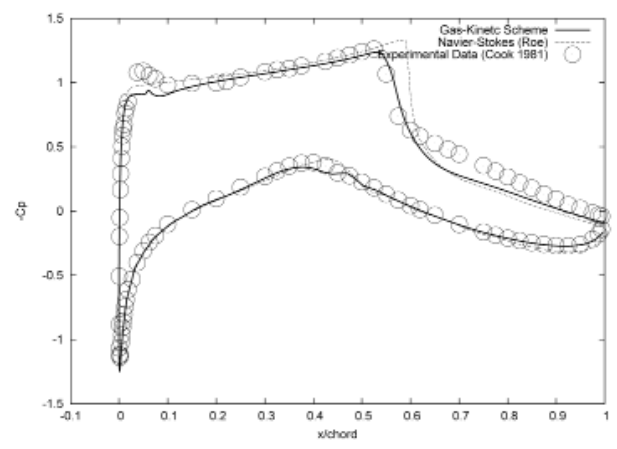

Figure 3. RAE2822 Case $10, \operatorname{Re}=6 \cdot 2106, M=$ $0 \cdot 745$, angle-of-attack $\alpha=2 \cdot 30^{\circ}$ pressure coefficient.

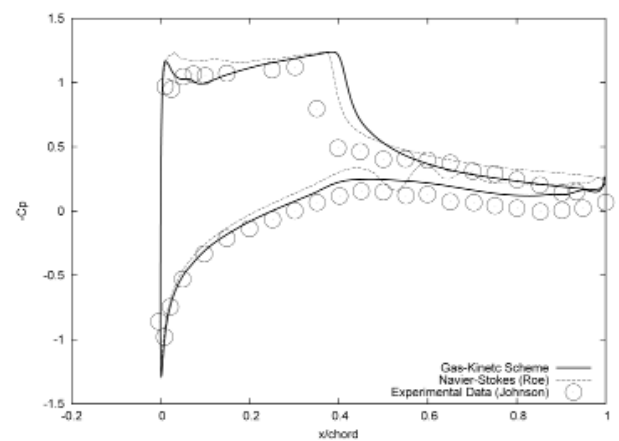

Figure 5. Aerofoil NACA 64A010, $\operatorname{Re}=2.0 \times 10^{6}, \mathrm{M}=$ $0 \cdot 75$, angle-of-attack $\alpha=6 \cdot 2^{\circ}$, pressure coefficient.

The results obtained from the NS and GKS schemes for Case 10 are shown in Fig. 3: the NS scheme predicts a shock at almost exactly $60 \%$ of the chord with a thickness of around $2 \%$, whereas the GKS predicts a shock position at $56 \%$ of the chord with a thickness of $6 \%$ of the chord. The GKS predictions are closer to the measurements by $\operatorname{Cook}^{(22)}$, which indicate a shock position at $55 \%$ with a thickness of $5 \%$ of the chord. The differences between the results provided by the two schemes are significant: in line with literature ${ }^{(2)}$, the shock position is predicted by the NS scheme with a $5 \%$ chord error, whereas the shock thickness is about three times smaller. Far smaller errors - about $1 \%$ chord - are predicted by the GKS scheme both in terms of shock position and thickness. The discrepancy that can be observed in the first $5 \%$ on the upper aerofoil side concerns the laminar-turbulent transition. A similar difference can also be observed in the literature ${ }^{(24)}$.

The results obtained from the NS and GKS schemes for the NACA 0012 case are shown in Fig. 4: the NS scheme predicts a shock at $55 \%$ of the chord with a thickness of around $2 \%$, whereas the GKS predicts a shock position at $51 \%$ of the chord with a thickness of approximately $5 \%$ of the chord. The GKS predictions are also in this case closer to the measurements by Harris ${ }^{(23)}$, which indicate a shock position at $50 \%$ with a thickness of approximately $3 \%$ of the chord. The differences between the results provided by the two schemes are consistent with those found in RAE2822 Case 10.

The poor prediction of separated flows by the $k-\omega$ model in NS schemes is well-known and due to the overprediction of turbulent shear stresses in the presence of incipient boundary layer 


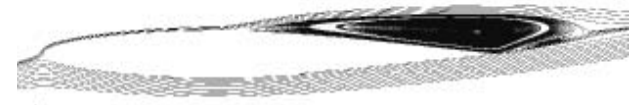

Figure 6. NACA 64A010, $\mathrm{Re}=2 \cdot 0 \times 10^{6}, \mathrm{M}=0 \cdot 75$, angle-of-attack $\alpha=6 \cdot 2$. Streamlines showing the large separation induced by the shock obtained from the NS solutions.

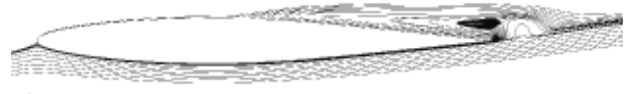

Figure 7. NACA 64A010, $\mathrm{Re}=2.0 \times 10^{6}, \mathrm{M}=0.75$, angle-of-attack $\alpha=6 \cdot 2$. Streamlines showing the large separation induced by the shock obtained from the the NS (left) and GKS (right) solutions. Details of streamlines around the trailing edge.
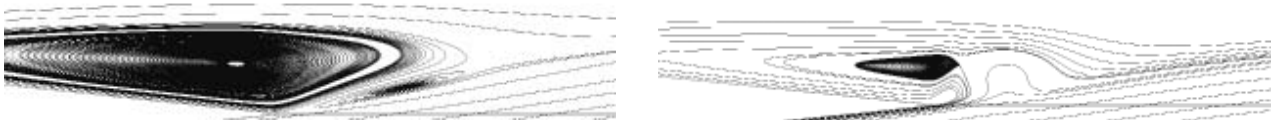

Figure 8. NACA 64A010, $\operatorname{Re}=2 \cdot 0 \times 10^{6}, M=0 \cdot 75$, angle-of-attack $\alpha=6 \cdot 2$. Streamlines showing the detail of the secondary flow around the trailing edge, obtained from the GKS solution.

separation: the errors found in this study from the NS scheme are consistent with the literature (refer for instance to Ref. 24 and references therein). Remarkably, the prediction obtained from the GKS scheme shows an accuracy comparable to the one provided by more sophisticated, higher-order turbulence models. A well-known member of this class of models is for instance the $k-\omega$ Explicit Algebraic Reynolds Stress Model (EARSM) model, proposed by Wallin and Johansson $^{(24)}$. It predicts the shock position in the RAE 2822 Case 10 case much more accurately than the standard $k$ - $\omega$ model $^{(24)}$.

\subsection{Aerofoil NACA 64A010 in transonic flow at high angle-of-attack}

The serie- 6 aerofoil NACA 64A010 has been investigated in transonic flow by Johnson ${ }^{(25)}$. The case $\operatorname{Re}=2 \cdot 0 \times 10^{6}, \mathrm{M}=0 \cdot 75$, angle-of-attack $a=6 \cdot 2$ has been considered here and investigated with the NS and GKS schemes. This flow case include a large separation with the shock wave located at about $30 \%$ of chord. The pressure coefficient is shown in Fig. 5. However, this case once more emphasises the differences in the vortex patterns predicted by the two schemes. The re-circulation area by the trailing edge is predicted to have two different patterns by the NS scheme (Fig. 6) and GKS (Fig. 7), especially around the trailing edge (Fig. 8). Interestingly, Johnson envisages re-circulation pattern qualitatively similar to the one predicted by the GKS scheme ${ }^{(25)}$.

Both schemes use second order reconstruction with van Leer limiter, approximate LU-SGS pre-conditioning and multi-grid. NS scheme uses Roe's approximate Riemann solver and GKS uses a bi-dimensional scheme. The computational meshes are $C$-type with $576 \times 128$ cells, and $y_{1}^{+}<1$.

\subsection{CONCLUSIONS}

Numerical schemes based on gas-kinetic theory are physically more consistent than those based on Navier-Stokes equations. In practical terms, in this study the numerical fluxes provided by the GKS are more accurate than the ones provided by a NS scheme, because the temporal and spatial evolution of the gas are coupled. The GKS is computationally more expensive but provides more accurate predictions in simulations involving complex flow patterns, such as vortexes and shock - boundary layer interaction. In turbulent flows, the GKS uses the information provided by 
a linear two-equation turbulence model differently to a NS scheme, without the need to specify any additional coefficients. Interestingly, the predictions provided by the GKS in benchmark cases involving a post shock separation are closer to experimental results than those provided by a well-known NS scheme. Comparable accuracy can be obtained with NS schemes if higher-order turbulence models are used instead of linear ones. However, these models are based on assumptions on the type of turbulence and formally not of general validity.

GKS are relatively new and far less known than NS schemes: the computational cost might be drastically reduced in the near future. The next steps include the sensitivity analysis to the type, order and multidimensionality of reconstruction, limiting, pre-conditioning and time integration.

\section{ACKNOWLEDGMENTS}

The author is grateful to $\mathrm{K}$. Xu for the information and the support provided.

\section{REFERENCES}

1. Cercignani, C. The Boltzmann equation and its applications, 1988, 67, Springer.

2. Godunov, S. Math. Sbornik 89, 1959, pp 271-306.

3. Chen, H., Kandasamy, S., Orszag, S., Shock, R., Succi, S. and Yakhot, V. Science, 2003, 301, pp 633-636.

4. Chen, H., Orszag, S., Staroselsky, I. and Succi, S. J Fluid Mech, 2004, 519, pp 301-314.

5. Righi, M. A gas-kinetic scheme for the simulation of turbulent flows, Rarefied Gas Dynamics, 2012.

6. Xu, K. J Comput Phys, 2001, 171, pp 289-335.

7. MaY, G. Srinivasan, B. and Jameson, A. J Comput Phys, 2007, 220, pp 856-878.

8. TANG, L. Comput Fluids, 2012, 56, pp 39-48.

9. Mandal, J. and Deshpande, S. Comput Fluids, 1994, 23, pp 447-478.

10. Chou, S. and Baganoff, D. J Comput Phys, 1997, 130, pp 217-230.

11. Xu, K. and K. Prendergast, J Comput Phys, 1994, 114, pp 9-17.

12. Bhatnagar, P., Gross, E. and Krook, M. Phys Rev, 1954, 94, p 511.

13. JAMESON, A. Schmidt, W. Turkel, E. ET AL, AIAA paper, 1981, 81, p 9.

14. WILcox, D. C. Turbulence Modeling for CFD, Third edition, 2006, DCW Industries, La Canada, CA, USA.

15. Yoon, S. and JAMESON, A. AIAA J, 1988, 26, pp 1025-1026.

16. JAMESON, A. Appl Math Comput, 1983, 13, pp 327-356.

17. Xu, K., Mao, M. and Tang, L. J Comput Phys, 2005, 203, pp 405-421.

18. Lee, H. and Huang, R. J Marine Science and Technology, 1998, 6, pp $29-37$.

19. JIANG, G. and Shu, C. Efficient implementation of weighted eno schemes, 1995, Tech report, DTIC Document.

20. Liu, X., Osher, S. and Chan, T. J Comput Phys, 1994, 115, pp 200-212.

21. LI, Q., Xu, K. and Fu, S. J Comput Physics, 2010, 229, pp 6715-6731.

22. Cook, P., McDonald, M. and Firman, M. AGARD Advisory, 1979.

23. Harris, C. NASA Technical Memorandum, 1981.

24. Wallin, S. and Johansson, A. J Fluid Mech, 2000, 403, pp 89-132.

25. Johnson, D., BAChalo, W. and Owen, F. NASA Technical Memorandum, 1981. 